\title{
Tratamento de carcinoma basoescamoso de ombro com a técnica de Tikhoff-Linberg modificada: um relato de caso
}

\author{
Treatment of basal squamous carcinoma of the shoulder with the modified Tikhoff-Linberg \\ technique: a case report
}
Tratamiento del carcinoma escamoso basal de hombro con la técnica de Tikhoff-Linberg modificada: reporte de un caso

Alan Pagoto Rossi ${ }^{1}$, Bruna Juliany Ferreira Cavalcante ${ }^{2}$, Caroline Ribeiro de Oliveira ${ }^{2}$, Hugo Marlon de Castro Negreiros ${ }^{1}$, Lázara Gabriela Oliveira Silva ${ }^{3 *}$, Raquel Freitas de Carvalho ${ }^{2}$, Monique Freire Santana ${ }^{1}$, Higino Felipe Figueiredo ${ }^{1}$, Manoel Jesus Pinheiro Coelho Júnior ${ }^{1}$.

\section{RESUMO}

Objetivo: Descrever a abordagem cirúrgica realizada no tratamento de paciente diagnosticado com carcinoma basoescamoso atendido em uma Instituição do Amazonas, evidenciando a eficácia e aplicabilidade da técnica de Tikhoff-Linberg modificada. Detalhamento do caso: Paciente masculino, 62 anos, com lesão ulcerada em ombro esquerdo, diagnosticado pela biópsia com basoescamocarcinoma gigante, submetido a cirurgia de amputação de ombro com a técnica Tikhoff-Linberg modificada, com amputação de ombro tipo III, com ressecção da clavícula e escápula, preservação da cabeça do úmero, linfadenectomia cervical e axilar, e utilização de retalho dermogorduroso deltopeitoral para cobertura da área cruenta. Sessenta dias após a cirurgia, o paciente apresentava movimentos da mão intactos e preservação de flexão e extensão do braço. O tratamento cirúrgico garantiu a ressecção completa da lesão, proporcionando uma boa recuperação com qualidade de vida para o paciente. Considerações finais: Não foi encontrado na literatura relatos semelhantes ao apresentado. Portanto, esse estudo contribuiu expressivamente nas pesquisas científicas levando em conta a raridade desse tipo de tumor, bem como a técnica cirúrgica selecionada para o tratamento, beneficiando profissionais na condução de casos semelhantes, além de benefícios adicionais aos pacientes.

Palavras-chave: Neoplasia, Carcinoma basoescamocelular, Ombro, Cirurgia oncológica.

\begin{abstract}
Objective: To describe the surgical approach performed in the treatment of basal squamous carcinoma attended at an institution in Amazonas, showing the effectiveness and applicability of the modified TikhoffLinberg technique. Case details: Male patient, 62 years old, with an ulcerated lesion on the left shoulder, diagnosed by biopsy with giant basoscamocarcinoma. He underwent shoulder amputation surgery with the modified Tikhoff-Linberg technique, with type III shoulder amputation - resection the clavicle and scapula, preservation of the humeral head, cervical and axillary lymphadenectomy, using deltopectoral dermofat flap to cover the open area. Thus, sixty days after surgery, the patient had intact hand movements and preserved arm flexion and extension. Surgical treatment ensured complete resection of the lesion, providing a good recovery with quality of life for the patient. Final considerations: No reports similar to the one presented were found in the literature. Therefore, this study significantly contributed to scientific research taking into account the rarity of this type of tumor, as well as the surgical technique selected for the treatment, benefiting professionals in the management of similar cases, in addition to additional benefits for patients.
\end{abstract}

Keywords: Neoplasm, Basosquamous carcinoma, Shoulder, Oncologic surgery.

\section{RESUMEN}

Objetivo: Describir el abordaje quirúrgico realizado en el tratamiento de carcinoma escamoso basal, asistió en una institución en Amazonas, mostrando la efectividad y aplicabilidad de la técnica modificada de Tikhoff-

${ }_{1}^{1}$ Fundação Centro de Controle de Oncologia do Estado do Amazonas (FCECON), Manaus - AM.

2 Faculdade Metropolitana de Manaus (FAMETRO), Manaus - AM.

3 Universidade Federal do Amazonas (UFAM), Manaus - AM. *E-mail: lazaragabi123@gmail.com 
Linberg. Datos del caso: Paciente masculino de 62 años con lesión ulcerada en hombro izquierdo, diagnosticado mediante biopsia de basoescamocarcinoma. Comprendió a cirugía de amputación de hombro con técnica modificada de Tikhoff-Linberg, con amputación de hombro tipo III - resección de clavícula y escápula, preservación de cabeza humeral, linfadenectomía cervical y axilar, utilizando colgajo dermofat deltopectoral para cubrir la zona abierta. Sesenta días después, el paciente tenía movimientos de la mano intactos y la flexión y extensión del brazo conservadas. El tratamiento quirúrgico aseguró la resección completa, proporcionando una buena recuperación con calidad de vida. Consideraciones finales: No se encontraron en la literatura reportes similares al presentado. Por tanto, este estudio contribuyó significativamente a la investigación científica teniendo en cuenta la rareza de este tipo de tumor, así como la técnica quirúrgica seleccionada para el tratamiento, beneficiando a los profesionales en el manejo de casos similares, además de beneficios adicionales para los pacientes.

Palabras clave: Neoplasia, Carcinoma basoescamoso, Hombro, Cirugía oncológica.

\section{INTRODUÇÃO}

O câncer de pele não melanoma é mundialmente o de maior prevalência dentre todas as neoplasias malignas, em ambos os sexos (BRAY F, et al., 2018). Contudo, se precocemente diagnosticado e adequadamente tratado apresenta-se com os menores índices de mortalidade (FERLAY J, et al., 2018).

Dados do Instituto Nacional De Câncer José Alencar Gomes Da Silva (INCA, 2019) indicam que no Brasil o câncer de pele não melanoma também é a neoplasia mais frequente, representando $30 \%$ de todos os tumores malignos do país. A estimativa é de que no país em cada ano de 2020 a 2022 ocorrerão 625 mil novos casos de câncer, sendo o de pele não melanoma o mais incidente com 177 mil, representando $27,1 \%$ de todos os casos de câncer em homens e $29,5 \%$ em mulheres.

Dentre os fatores de risco para essa patologia, destacam-se pele clara, faixa etária acima dos 40 anos, história pessoal ou familiar deste câncer ou de doenças cutâneas prévias e principalmente a exposição prolongada ao sol, radiação ultravioleta (UV), principalmente na infância e na adolescência, e exposição a câmeras de bronzeamento artificial. Porém, esse perfil de idade vem se modificando e reduzindo devido à constante exposição dos jovens aos raios solares (INCA, 2019).

Os dois tipos histológicos de tumores de pele não melanoma mais frequentes são o Carcinoma Basocelular (CBC) mais prevalente e o menos agressivo, e o Carcinoma Escamocelular (CEC). O Carcinoma Basoescamoso (CBE) ou metatípicos apresentam características histológicas de células basais e escamosas interligadas por uma área de transição (AMERICAN CANCER SOCIETY, 2019).

A primeira descrição do carcinoma basoescamoso foi feita em 1910 por MacCormac, destacando seu comportamento biológico localmente mais agressivo e potencialmente metastático. Possuem incidência de cerca de $2 \%$ dentre todos os tumores de pele, frequentemente observados em região de cabeça e pescoço (GUALDI G, et al., 2021).

O diagnóstico histopatológico, bem como a descrição de fatores prognósticos, baseia-se nos achados morfológicos encontrados nas resseções completas. Além disso, o comprometimento das margens cirúrgicas é um importante fator prognóstico, assim como o comprometimento linfonodal. Sendo a cirurgia com completa ressecção do tumor o tratamento de eleição (JORDAN WO, et al., 2018).

A cirurgia de Tikhoff-Linberg é uma ressecção interescapulotorácica com preservação do membro, uma opção cirúrgica para tumores ósseos e de partes moles do úmero proximal e da cintura escapular. O tipo II dessa cirurgia é nomeada de Tikhoff-Linberg modificada ou escapulectomia parcial medial, a qual preserva a maior parte da cintura escapular (SILVA GS, et al., 2019).

Relatamos no presente artigo a abordagem cirúrgica de um caso de carcinoma basoescamoso de ombro em um paciente masculino de 62 anos, atendido em um hospital oncológico terciário no Amazonas, evidenciando a eficácia e a aplicabilidade da técnica de Tikhoff-Linberg modificada para casos semelhantes ao descrito. 


\section{DETALHAMENTO DO CASO}

Os dados deste relato foram coletados de informações autorizadas pelo paciente, mediante ao Termo de Consentimento Livre e Esclarecido (TCLE).

Paciente F.C.B., masculino, 62 anos, diabético, branco, mecânico, natural do Rio de Janeiro, residente em Manaus. Apresentava na primeira consulta, em fevereiro de 2019, lesão ulcerada de $7 \mathrm{~cm}$ em região clavicular esquerda há mais de um ano. Submetido a tomografia computadorizada (TC) de tórax e abdome que não evidenciaram doença metastática, porém, evidenciaram comprometimento ósseo em clavícula. Submetido a biópsia excisional, com diagnóstico preliminar de CEC. A abordagem cirúrgica proposta foi de amputação de ombro com a técnica Tikhoff-Linberg modificada.

O paciente permaneceu afastado por um ano e retornou para nova consulta, em fevereiro de 2020, apresentando progressão importante da lesão para aproximadamente $10 \mathrm{~cm}$ de extensão (Figura 1, A). Relata que não aceitou o tratamento inicialmente devido ao risco de amputação do membro superior esquerdo, com consequente perda funcional, ficando impedido de pilotar sua motocicleta. Foram solicitados novos exames para estadiamento que não evidenciaram doença à distância e nem linfonodomegalia.

Foi submetido a amputação de ombro tipo III (ressecção da clavícula e escápula), com preservação da cabeça do úmero, linfadenectomia cervical nível I, II, III e IV e linfadenectomia axilar, utilizado retalho dermogorduroso deltopeitoral para cobertura da área cruenta (Figura 1, B, C e D).

Figura 1 - Achados intraoperatórios



Legenda: A: Lesão ulcerada infiltrativa em ombro esquerdo; B: Visão craniocaudal após ressecção; C: Aspecto final da cirurgia, visão antero-posterior; D: Aspecto final da cirurgia, visão frontal; E: Paciente após 60 dias de procedimento. Fonte: Rossi AP, et al., 2021. 
O exame histopatológico da peça cirúrgica evidenciou um Carcinoma basoescamoso gigante e ulcerado (Figura 2: A, B e C), medindo 10,5 x 3,5 cm, com comprometimento ósseo (Figura 2: D) e muscular (Figura 2: E), com invasão perineural. Não foram observadas invasão angiolinfática, comprometimento de margens ou dos sete linfonodos examinados.

Figura 2 - Achados anatomopatólogicos

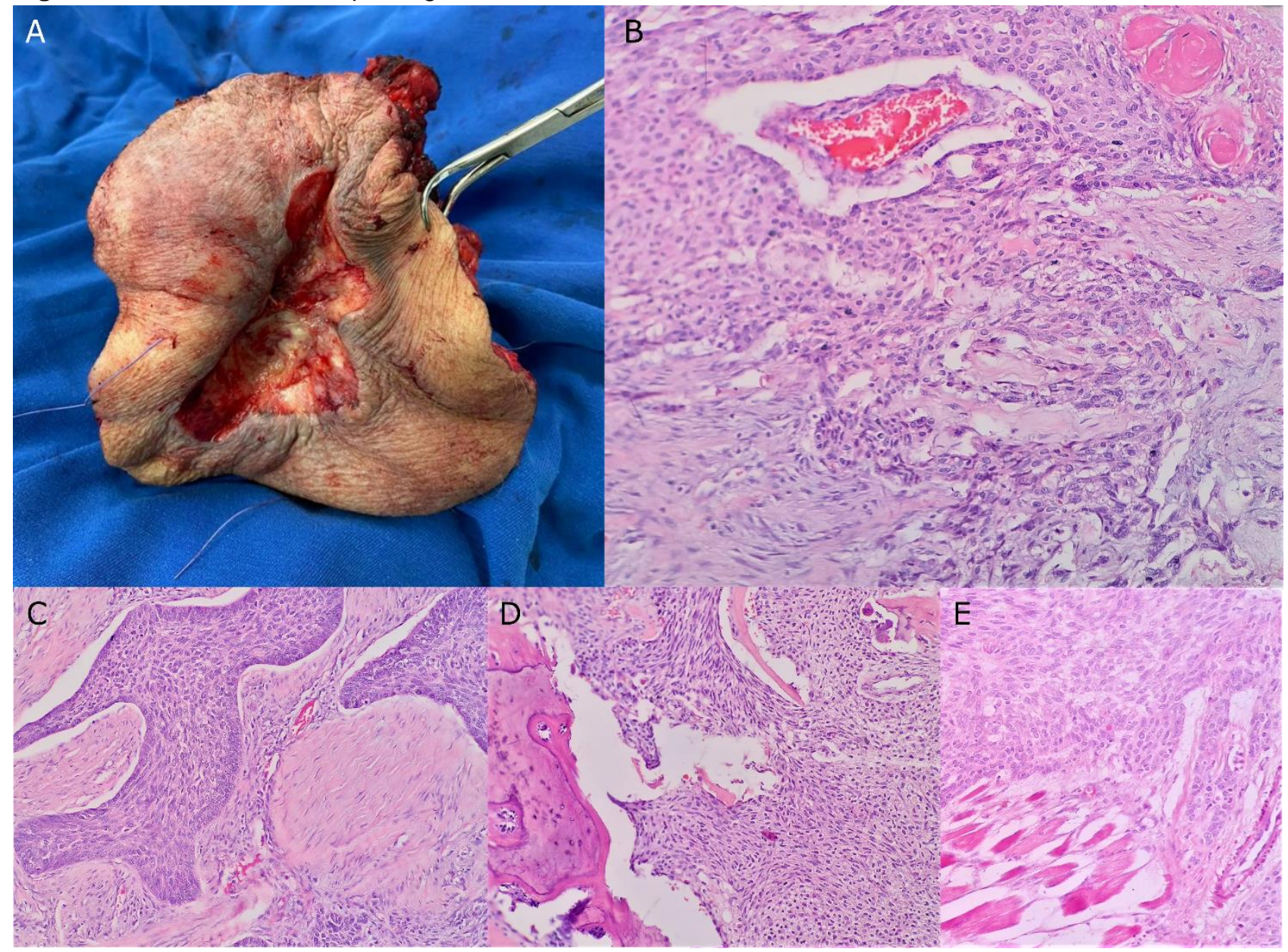

Legenda: A: Extensa lesão ulcerada, com fundo cruento, de bordas regulares; B: Células arredondadas, de escasso citoplasma, formando pérolas córneas em áreas focais, em componente escamoso; C: Células arredondadas, de escasso citoplasma, formando ninhos irregulares, com paliçada periférica e artefatos de retração, em componente basaloide; D: Invasão em fragmentos ósseos; E: Infiltração em músculo esquelético. Fonte: Rossi AP, et al., 2021.

O serviço de oncologia clínica contraindicou tratamento adjuvante devido a ausência de comprometimento linfonodal. Paciente recebeu alta no quinto dia de pós-operatório, apresentando deiscência parcial do retalho, tratada com curativos, permanecendo com tipóia por 60 dias. Após a retirada da mesma, o paciente apresentava movimentos da mão intactos e preservação de flexão e extensão do braço, em reabilitação para capacidade funcional desejada, de pilotar motocicleta (Figura 1: E).

\section{DISCUSSÃO}

CBE é definido como um tumor que possui características histopatológicas de carcinoma de células escamosas, mostrando áreas de transição de basaloide a células escamosas, ou células de diferenciação mista. Esta morfologia tem sido referida pelos nomes de Carcinoma basoescamoso, carcinoma basocelular metatípico, carcinoma basocelular com diferenciação escamosa e carcinoma basocelular ceratótico (TAN C, et al., 2017). 
Chiang A, et al. (2019), relataram após estudos realizados, a existencia de fatores genéticos para o surgimento do CBE. Identificaram a via Hedgehog como o principal mecanismo ligado a patogenicidade, encontrada em $45 \%$ dos tumores, corroborando para a hipótese de que um CBE deriva de um CBC, entretanto, com maior agressividade local e potencial metastático.

Alcala DP, et al. (2017), relatou que a topografia mais encontrada de lesões correspondentes ao carcinoma basoescamoso são de regiões foto expostas, como a cabeça e o pescoço, que representam de 82 a $97 \%$ dos casos, e menos comumente em pálpebras, pescoço, tronco e membros, como no caso descrito, em região deltoidea.

Zaleta LB, et al. (2021), em relato de caso apresentou paciente masculino, branco de 53 anos com carcinoma basoescamoso em região malar esquerda. As características físicas do paciente assemelham-se ao do paciente do presente artigo nos fatores de risco desse tipo de câncer, sendo eles: homens, brancos, maiores de 40 anos. Ambos os pacientes, de Zaleta LB et al. (2021) e deste relato, apresentavam o diagnóstico histopatológico prévio da lesão como CEC e após exérese completa da lesão foi possível definir como CBE. Dessa forma, o diagnóstico do carcinoma basoescamoso é realizado através de exame histológico, preferencialmente da peça cirúrgica, devido ao risco de não se evidenciar as duas apresentações morfológicas, já que biópsias superficiais e de fragmentos não atingem índices de diagnósticos suficientes (ZALETA LB, et al., 2021).

Elmahi $\mathrm{H}$, et al. (2016), relata que ainda não existe protocolo para conduta ou abordagem padronizada para os tumores de pele não melanomas do tipo carcinoma basoescamoso (CBE), devido à ausência de informações e pesquisas disponíveis na literatura científica sobre esse tema. Contudo, até então, a abordagem cirúrgica tem sido o tratamento de escolha.

Embora, ainda não haja definição quanto a margem cirúrgica necessária, recomenda-se que na ressecção de lesões com diâmetro inferior a $2 \mathrm{~cm}$ a margem seja de 4 a $6 \mathrm{~mm}$. Contudo, em tumores maiores que 2; carcinomas basocelulares com recidiva; subtipos: esclerodermiforme, infiltrativo, micronodular e basoescamoso; localizados na zona $\mathrm{H}$, opta-se pela realização da cirurgia micrográfica de Mohs, considerada padrão-ouro para muitos autores (TOLKACHJOV SN, et al., 2017).

Tan C, et al. (2017), descreveu que a recorrência local desse tumor, posterior à excisão ampla, é de até $45 \%$, valor duas vezes maior que dos carcinomas basocelular e escamocelular. Bem como, o tamanho tumoral é uma condição preditiva para surgir novamente. A prevalência de recorrência é local, seguinte temse metástase linfonodal e parcamente prevalente relata-se metástase pulmonar. Para tumores maiores que $3 \mathrm{~cm}$ se nota índice metastático de $2 \%$.

Além da abordagem cirúrgica, em alguns casos opta-se também pela radioterapia, quando houver margens cirúrgicas comprometidas e a nova abordagem possa ocasionar comprometimento estético ou funcional. Assim como os tumores recidivos e metastáticos que possuem pior prognóstico, podem também ser abordados com quimioterapia, sendo essa feita através do uso de terapia alvo. Nesse sentido, drogas como o vismodegib e sonidegib são consideradas promissoras e seguem em estudos para uso em casos de carnicinomas basoescamosos avançados e inoperáveis (AGUIAR D, et al., 2017)

A invasão perineural é uma via independente de disseminação tumoral, não sendo comum esse acometimento em tumores de pele, como o CBE. Entretanto, nota-se a associação da presença de invasão perineural a tumores de pior prognostico e de maiores índices de morbimortalidade, bem como quando há comprometimento linfonodal (SANTOS ABO, et al., 2017).

Shehadeh A, et al. (2019), exibiu a classificação criada em 1991 por Malaver de ressecções da cintura escapular, a qual divide-se em 6 tipos, sendo eles: Tipo I - Ressecção umeral proximal intra-articular; Tipo II - Escapulectomia parcial; Tipo III - Escapulectomia total intra-articular; Tipo IV - Ressecção extra-articular da escapula e cabeça do úmero; Tipo V - Ressecção de glenóide e umeral extra-articular; Tipo VI Ressecção umeral e escapular total e extra-articular. A cirurgia proposta para a maioria dos tumores com comprometimento escapuloumeral é de amputação interescapulotorácica. Contudo, há a opção de procedimentos modificados mais conservadores tendo como vantagem a preservação do membro, 
principalmente para casos de tumores malignos dessa região, os quais não apresentem infiltração de feixe vasculonervoso principal ou da parede torácica (SHEHADEH A, et al., 2019). Em complemento à essa literatura, na abordagem do caso relatado nesse presente estudo, optou-se pela mudança na estratégia cirúrgica com a preservação da cabeça do úmero e ressecção completa da clavícula e escápula, sem a utilização de prótese.

Serrano PM, et al. (2018), em concordância ao estudo anterior relatado, descreve que para a abordagem de tumores do ombro ou cintura escapular, na maioria das vezes a amputação interescapulotorácica como exclusivo tratamento cirúrgico radical. Todavia, nos casos em que o tumor não abrange o feixe neurovascular a cirurgia de Tikhoff-Linberg é a opção para garantir a preservação do membro envolvido. Nota-se que embora haja altas taxas de complicações globais relacionadas a essa abordagem, ainda assim, demonstrou ser uma conduta plausível para os tumores extensos da região do ombro, evitando prejuízos funcionais, estéticos e psicológicos.

Silva GS, et al. (2019), em seu relato de caso, descreveu conduta elaborada por equipe multidisciplinar para tratamento de sarcoma histiocítico ósseo localizado em escápula. Inicialmente a conduta foi com quimioterápicos e posterior abordagem com técnica cirúrgica modificada de Tikhoff-Linberg tipo II, a qual baseou-se na retirada do tumor associado a amputação parcial da escapula direita, seguido por mais um ciclo com quimioterápicos e assistência da fisioterapia. Notou-se então relevante melhora nos aspectos funionais do membro que tinha o tumor e foi abordado.

Serrano PM, et al. (2018), explica que na cirurgia de Tikhoff-Linberg, para abordagem dos tumores de cintura escapular, há comumente a ressecção do terço distal da clavícula e a ressecção da cabeça do úmero, podendo ou não ser utilizada uma prótese metálica para fixação do úmero remanescente. Em contrapartida, no caso descrito, a cirurgia realizada para esse tipo de tumor raro de pele (basoescamoso) com comprometimento ósseo, foi associada à linfadenectomia ampla e ressecção extensa de tecidos moles, com necessidade de retalho, nesse caso, dermogorduroso do peitoral, que não são usuais na cirurgia clássica. Além disso, houve também a preservação total da cabeça do úmero e ressecção completa da clavícula.

A abordagem de tumores malignos da cintura escapular sempre foi um grande desafio. Nota-se também, recorrentes complicações da abordagem com amputação, sendo as principais seroma, deiscência, neuroma ou dor fantasma. Dessa forma, em casos de tumores sem invasão de feixe neurovascular opta-se por abordagens em que haja ressecções mais conservadoras. Nesse sentido, Tikhoff realizou de forma inédita em 1922 a ressecção interescapulotorácica para tumores de partes moles da cintura escapular, e posteriormente, em 1928, Linberg descreveu novos casos de utilização dessa técnica. A partir de então, nomeou-se como técnica cirurgica de Tikhoff-Linberg (SERRANO PM, et al., 2018).

Considera-se, portanto, que não foi encontrado nenhum relato semelhante a conduta do caso exposto com a realização da cirurgia de Tikhoff-Linberg para tumores de pele, do tipo basoescamoso, e se mostra uma alternativa para casos avançados, em que a necessidade de amputação do membro é considerada. Mesmo não sendo o tratamento de primeira escolha, nesse caso, a abordagem cirúrgica realizada resultou na ressecção completa da lesão, proporcionando boa recuperação e qualidade de vida para o paciente. Destacam-se a técnica cirúrgica selecionada para o tratamento de um CBE, conhecidamente de comportamento biológico mais agressivo, beneficiando profissionais e pacientes na condução de casos semelhantes.

\section{REFERÊNCIAS}

1. AGUIAR D, et al. Tratamento cirúrgico de carcinoma basocelular do tipo esclerodermiforme e recidiva linfonofal: relato de caso. SEMPESq -Semana de Pesquisa da Unit, 2017; 5.

2. ALCALÁ DP, et al. Carcinoma basocelular metatípico: el gran simulador. Rev Cent Dermatol Pascua, 2017; 26(3):1003.

3. BRAY F, et al. Global cancer statistics 2018: GLOBOCAN estimates of incidence and mortality worldwide for 36 cancers in 185 countries. CA: a cancer journal for clinicians - Hoboken, 2018; 68(6): 394-424.

4. CARDOSO P, et al. Intraosseous synovial sarcoma of the body of the scapula. Imaging Med, 2016; 8: 49-51. 
5. ELMAHI H, et al. L'apport de la dermoscopie dans le carcinome basosquameux. Pan African Med J, 2016; 25:252.

6. FERLAY, J. et al. Estimating the global cancer incidence and mortality in 2018: GLOBOCAN sources and methods. International journal of cancer, 2019; 144(8): 1941- 1953.

7. GUALDI G, et al. Characterization of Basosquamous Cell Carcinoma: A Distinct Type of Keratinizing Tumour. Acta Derm Venereol, 2021; 101(1):4.

8. HOORENS I, et al. Mohs micrographic surgery for basal cell carcinoma: evaluation of the indication criteria and predictive factors for extensive subclinical spread. Br J Dermatol, 2016; 174(4):847- 52.

9. INSTITUTO NACIONAL DE CÂNCER JOSÉ ALENCAR GOMES DA SILVA. Tipos de câncer. Rio de Janeiro: INCA, 2019; 49p.

10. JORDAN WO, et al. Basosquamous Carcinoma: A Single Centre Clinicopathological Evaluation and Proposal of an Evidence-Based Protocol. Journal of Skin Cancer, 2018; 7p.

11. LUENGO FLM, et al. Miasis cutánea asociada a carcinoma basoescamoso: reporte de un caso por Cochliomyia hominivorax. Dermatología Cosmética, Médica y Quirúrgica, 2019; 17 (4).

12. SANTOS ABO, et al. Quais características dos carcinomas estão associados a invasão perineural?. Braz. j. otorhinolaryngol., 2017; 83 (1).

13. SCOTT J, et al. Treatment of inoperable basosquamous carcinoma with hedge-hog inhibitors, vismodegib and sonedigib: A case series. Journal Of The American Academy Of Dermatology, 2019; 81(4): 360.

14. SERRANO PM, et al. Procedimento de tikhoff-linberg - relato de casos. Rev Port Ortop Traum, 2018; 26(2): $166-174$.

15. SILVA GS, et al. Sarcoma histiocítico ósseo em escápula: relato de caso. Rev. bras. ortop., 2019; 54:1.

16. SILVEIRA JG, et al. Desenvolvimento de protocolos institucionais para o tratamento de carcinomas de queratinócitos por excisão cirúrgica e avaliação de margens profundas e laterais por biópsia intraoperatória de congelação. Dissertação (Mestrado em Ciências) - Faculdade de Ciências Médicas da Universi dade Estadual de Campinas, Campinas, 2020; 104p.

17. SHEHADEH A, et al. Shoulder girdle resection: surgical technique modification and introduction of a new classification system. World Journal of Surgical Oncology, 2019; 17: 107.

18. TAN C, et al. Basosquamous carcinoma: controversy, advances, and future directions, Dermatol Surg 2017; 43(1):2331.

19. TOLKACHJOV SN, et al. Understanding Mohs Micrographic Surgery: A Review and Practical Guide for the Nondermatologist. Mayo Clin Proc., 2017; 92(8):1261-1271.

20. ZALETA LB, et al. Carcinoma basoescamoso. A propósito de un caso y la importancia de la revisión clínica y dermatoscópica. Dermatología Cosmética, Médica y Quirúrgica, 2021; 19(1):16-19. 